\title{
Erratum to: JMJD2A-dependent silencing of Sp1 in advanced breast cancer promotes metastasis by downregulation of DIRAS3
}

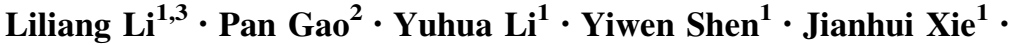

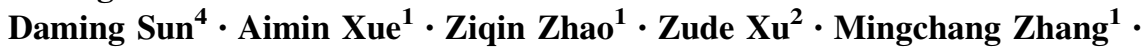 \\ Beixu $\mathbf{L i}^{1} \cdot$ Jieqing Jiang ${ }^{1}$
}

Published online: 23 February 2016

(C) Springer Science+Business Media New York 2016

\section{Erratum to: Breast Cancer Res Treat (2014) \\ 147:487-500 \\ DOI 10.1007/s10549-014-3083-7}

In the original publication of the article, the Fig. 5 was published erroneously with mislabeling of the downregulating protein and duplication of an image in Fig. 5e. The corrected Fig. 5 shown below is the correct representation of the data from this study. The authors apologize for this error.

The online version of the original article can be found under doi:10.1007/s10549-014-3083-7.

Aimin Xue

amxue@fudan.edu.cn

Ziqin Zhao

zqzhao@shmu.edu.cn

Liliang Li

11111010016@fudan.edu.cn

Pan Gao

11111010014@fudan.edu.cn

Yuhua Li

12111010086@fudan.edu.cn

Yiwen Shen

shenyiwen@fudan.edu.cn

Jianhui Xie

jhxie@fudan.edu.cn

Daming Sun

daming1575@126.com

Zude Xu

zdxu@shmu.edu.cn

Mingchang Zhang

071101048@fudan.edu.cn

Beixu Li

09111010011@fudan.edu.cn
Jieqing Jiang

jqjiang@shmu.edu.cn

1 Department of Forensic Medicine, School of Basic Medical Sciences, Fudan University, 138 Yixueyuan Road, Xuhui District, Shanghai 200032, People's Republic of China

2 Department of Pathology, School of Basic Medical Sciences, Fudan University, 138 Yixueyuan Road, Xuhui District, Shanghai 200032, People's Republic of China

3 Division of Forensic Pathology, University of Maryland School of Medicine, 900 West Baltimore Street, Baltimore, MD 21223, USA

4 Forensic Science Center, East China University of Political Science and Law, 112 Huayang Road, Changning District, Shanghai 200042, People's Republic of China 
$\mathbf{a}$

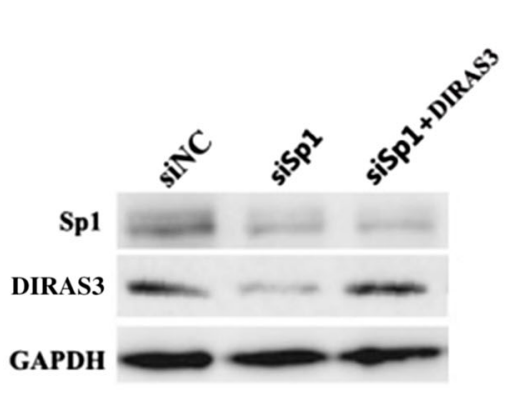

c

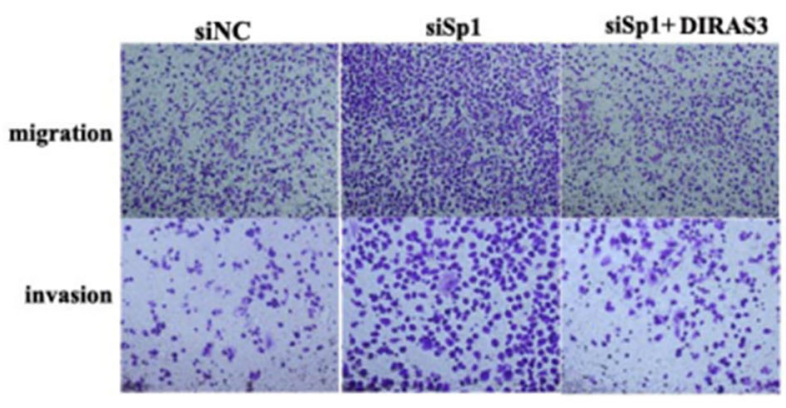

e

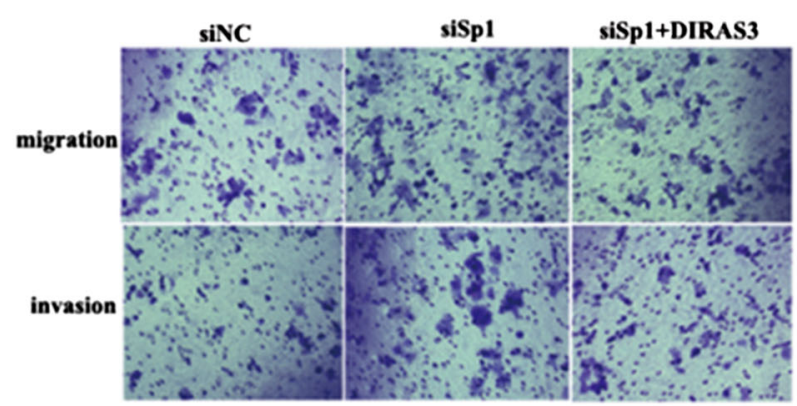

Fig. 5 Re-expression of DIRAS3 reversed Sp1 knockdown-mediated breast cancer cell migration and invasion. a Protein levels of Sp1 and DIRAS3 in MDA-MB-231 cells were assessed by Western blot analysis after plasmid and/or siRNA transfection. Transfection of siSp1 led to decrease in Sp1 expression as well as DIRAS3 expression. Additionally, transfection of DIRAS3 plasmid into Sp1silenced MDA-MB-231 cells efficiently elevated DIRAS3 expression. GAPDH was used as a loading control. b MDA-MB-231 cells with distinct treatments were subjected to wound-healing assay. Representative photographs at $24 \mathrm{~h}$ were taken (left panel). Magnification: $\times 100$. Percentage wound recovery was also shown (right panel). $* P<0.05$. c MDA-MB-231 cells with distinct treatments were
MDA-MB-231

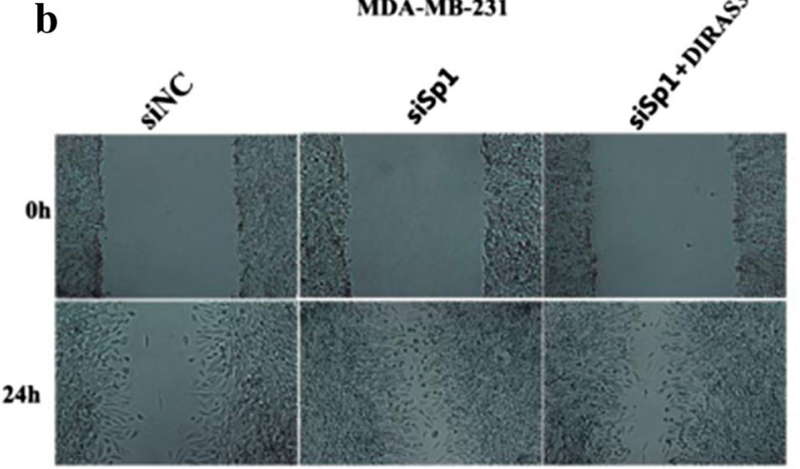

d
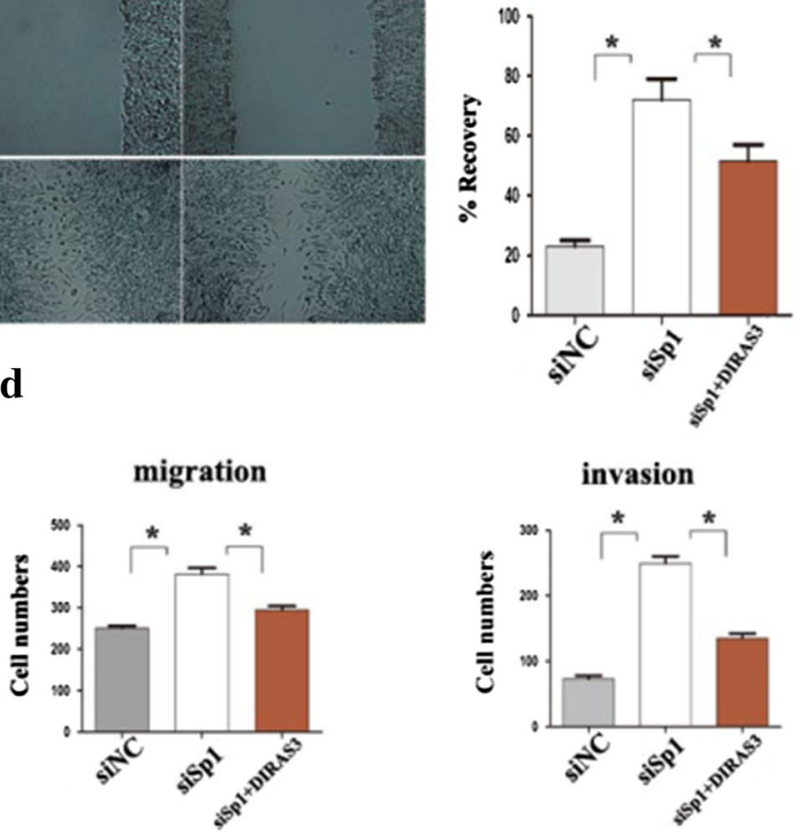

f

migration
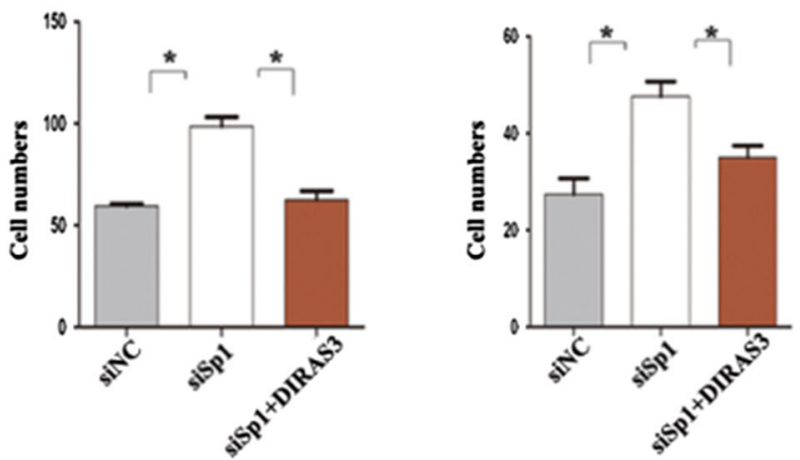

subjected to transwell migration and invasion assays. $12 \mathrm{~h}$ post culture, cells were stained with crystal violet. Representative photographs from each group were taken for subsequent cell number counting. Magnification: $\times 200$. d Migratory and invasive MDA-MB231 cells were counted and shown. Cell numbers represented average values from three microscopic visions of each group. ${ }^{*} P<0.05$. e, $\mathbf{f}$ In MCF-7 cells, re-expression of DIRAS3 was also observed to reverse $\mathrm{Sp} 1$ knockdown-mediated breast cancer cell migration and invasion, as revealed by the crystal violet staining (e) and transmigrated cell number counting (f). Magnification: $\times 200$. ${ }^{*} P<0.05$. siNC, negative control siRNA 